\title{
Survival Analysis of Factors Influencing Cyclic Fatigue of Nickel-Titanium Endodontic Instruments
}

\author{
Eva Fišerová, ${ }^{1}$ Martina Chvosteková, ${ }^{1}$ Silvie Bělašková, ${ }^{1,2}$ \\ Michal Bumbálek, ${ }^{3,4}$ and Zdeněk Joska ${ }^{5}$ \\ ${ }^{1}$ Department of Mathematical Analysis and Applications of Mathematics, Faculty of Science, Palacký University in Olomouc, \\ 17 Listopadu 12, 77146 Olomouc, Czech Republic \\ ${ }^{2}$ Department of Mathematics, Faculty of Applied Informatics, Tomas Bata University in Zlín, Nám. T.G. Masaryka 5555, \\ 76005 Zlin, Czech Republic \\ ${ }^{3}$ Institute of Clinical and Experimental Dental Medicine, First Faculty of Medicine, \\ Charles University General Teaching Hospital, Prague, Czech Republic \\ ${ }^{4}$ Onedent, Př́kop 8, 60200 Brno, Czech Republic \\ ${ }^{5}$ Communication and Information Systems Agency, Czech Army, Tychonova 1, 16001 Prague, Czech Republic
}

Correspondence should be addressed to Martina Chvosteková; martina.chvostekova@upol.cz

Received 27 May 2015; Accepted 9 September 2015

Academic Editor: Luigi Nicolais

Copyright (C) 2015 Eva Fišerová et al. This is an open access article distributed under the Creative Commons Attribution License, which permits unrestricted use, distribution, and reproduction in any medium, provided the original work is properly cited.

\begin{abstract}
Objective. The aim of this study was to validate a survival analysis assessing the effect of type of rotary system, canal curvature, and instrument size on cyclic resistance. Materials and Methods. Cyclic fatigue testing was carried out in stainless steel artificial canals with radii of curvature of 3 or $5 \mathrm{~mm}$ and the angle of curvature of 60 degrees. All the instruments were new and $25 \mathrm{~mm}$ in working length, and ISO colour coding indicated the instrument size (yellow for size 20; red for size 25). Wizard Navigator instruments, Mtwo instruments, ProTaper instruments, and Revo-S instruments were passively rotated at 250 rotations per minute, and the time fracture was being recorded. Subsequently, fractographic analysis of broken tips was performed by scanning electron microscope. The data were then analysed by the Kaplan-Meier estimator of the survival function, the Cox proportional hazards model, the Wald test for regression covariates, and the Wald test for significance of regression model. Conclusion. The lifespan registered for the tested instruments was Mtwo $>$ Wizard Navigator $>$ Revo-S $>$ ProTaper; $5 \mathrm{~mm}$ radius $>3 \mathrm{~mm}$ radius; and yellow $>$ red in ISO colour coding system.
\end{abstract}

\section{Introduction}

Nickel-titanium (Ni-Ti) instruments are increasingly used in the present endodontics due to the outstanding combination of mechanical properties superior to those of stainless steel. Yet, despite increased flexibility, an unexpected failure during root canal treatment is still a matter of great concern, as these instruments can undergo fracture within their elastic limit without any visible sign of previous permanent deformation [1-3]. The fracture of rotary Ni-Ti instruments can occur as a consequence of torsional overload or as a result of flexural fatigue [3,4]. Clinically, Ni-Ti rotary instruments are subjected to both torsional load and cyclic fatigue [5]. Torsional fracture occurs when the tip of the instrument is stuck in a canal while the rest of the tool is being rotated. When the elastic limit of the metal is exceeded by the torque exerted by the handpiece, a fracture of the tip becomes inevitable [6]. The cyclic fatigue fracture is caused by alloy fatigue. The instrument freely rotates in a curved canal, generating tensions/compressions cycles at the point of maximum flexure until the fracture occurs $[2,5,7]$. Cyclically fatigued instruments show no macroscopic evidence of plastic deformation, but instruments that fracture as a result of torsional overload demonstrate variable deformation, such as unwinding, straightening, reverse winding, and twisting [3, 8]. The incorrect clinical use [9] and several other factors contribute to instrument fracture $[10]$. 
There are currently many rotary endodontic systems of various designs and dimensions, which are used for cleaning and shaping of root canals. The development of engine-driven rotary endodontic instruments, along with the absence of adequate testing instrument standards, necessitates further analysis in all areas. The resistance of rotary instruments to cyclic fatigue, for instance, is affected by the angle and radius of canal curvature. Instrument lifespan also decreases with the increased severity in the angle and the radius of the curves around which the instrument rotates [1, 2, 7]. Testing instruments in canals with radii of curvature of 2, 5, and $10 \mathrm{~mm}$ showed that the smaller the radius, the shorter the life of the instrument when rotating. The resistance of rotary instruments to cyclic fatigue is also affected by the diameter of the instrument. Several studies have shown that an increased diameter, which is determined by the tip size and taper, reduces the time to fracture at the point of maximum curvature of the instrument $[1,2,7]$. The fatigue resistance caused by rotational speed in metal-simulated canals was studied for instruments of sizes 30 and 40, and it was concluded that the speed is not a significant factor [2]. Although the morphology of a Ni-Ti rotary file on its performance has been investigated [11-14], the influence of file design on the cyclic fatigue stress remains unclear, so the effect of a Ni-Ti rotary system during root canal treatment still necessitates further study.

The cyclic fatigue resistance is traditionally analysed by one-way ANOVA. The time to failure is multiplied by rotation per minute (rpm) in order to obtain the number of cycles to failure. In this paper, we analyse fatigue resistance from a different point of view. Survival analysis is a collection of statistical procedures for analysing the duration until the occurrence of an event of interest, the event in this study being the cyclic fatigue fracture of a rotary file. In the survival approach, Ni-Ti instruments are compared by analysing their times to failure. The survival analysis approach allows us to estimate the probability that failure of rotary system does not occur beyond a specific time and to assess the hazard function, which gives the instantaneous potential per unit time for the failure to occur, provided that the instrument has survived up to that time.

The data-set comes from the Department of Mechanical Engineering at the University of Defence in Brno, where the cyclic fatigue resistance of four $\mathrm{Ni}-\mathrm{Ti}$ rotary systems was tested. This study assesses the times to failure in relation to the type of Ni-Ti rotary system (ProTaper, Revo-S, Wizard Navigator, and Mtwo), radius of curvature to which the instruments were subjected during preparation ( 3 and $5 \mathrm{~mm}$ ), and instrument size identified by ISO standards (yellow for size 20 and red for size 25).

\section{Survival Analysis of Cyclic Fatigue Lifetime of the Rotary System/Statistical Background}

The primary interest is usually given to the survival function, also known as reliability function, which is the probability that the time of the cyclic fatigue fracture is longer than a specified time $t$. In other words, the survival function indicates the probability that the rotary system resists beyond a specified time. The Kaplan-Meier estimator [15], also known as the product-limit, is a well-known estimator for the survival function. The Kaplan-Meier estimator of the survival function is

$$
\widehat{S}(t)=\prod_{i: t_{(i)}<t}\left(1-\frac{d_{i}}{n_{i}}\right),
$$

where $t_{(1)}<t_{(2)}<\cdots<t_{(m)}$ denote the $m$ ordered distinct failure times of the fracture due to cyclic fatigue in a group of instruments used when a fixed category was set (e.g., all instruments rotated in stainless-steel blocks with artificial canals with a $3 \mathrm{~mm}$ radius of curvature), $d_{i}$ is the number of the cyclic fatigue fractures at a time $t_{(i)}$, and $n_{i}$ is the number of the cyclic fatigue fractures before the time $t_{(i)}$. The Kaplan-Meier estimate is a step function with the points of discontinuity (jumps) at the observed times of the cyclic fatigue fractures.

The most widely used method to investigate several variables at a time is the Cox proportional hazards model [16]. The hazard function at a time $t$, also known as the failure function, is the probability that, during a very short time interval, an event will occur, conditional on not having the event up to a time $t$. The hazard function is nonnegative; it assesses the instantaneous risk of the cyclic fatigue fracture for a rotary system which has resisted a time $t$. The Cox proportional hazards model specifies the hazard function to covariates for individual instruments. In our study, the hazard function is related to the radius of curvature, type of $\mathrm{Ni}$ Ti rotary system, and instrument size, which are categorical variables. We distinguish two categories for the radius (3 and $5 \mathrm{~mm}$ ), four categories for the type (Mtwo, Wizard Navigator, Revo-S, and ProTaper), and two categories for the instrument size (ISO colour coded yellow and red). For each categorical variable one category is excluded and is considered as the reference category. Our selection includes a $3 \mathrm{~mm}$ radius, Wizard Navigator, and red ISO colour. When modelling with the categorical variables, the so-called dummy variables (indicators of categories) are usually used; for example, $I_{\text {[ProTaper }]}$ denotes a dummy variable coded 1 for all ProTaper instruments and 0 for the other systems. The model can thus be formally expressed as

$$
\begin{aligned}
& h(t, \mathbf{x})=h_{0}(t) \exp \left(\beta_{1} I_{[5 \mathrm{~mm}]}+\beta_{2} I_{[\text {ProTaper }]}+\beta_{3} I_{[\text {Mtwo }]}\right. \\
& \left.\quad+\beta_{4} I_{[\text {Revo-S }]}+\beta_{5} I_{[\text {yellow }]}\right),
\end{aligned}
$$

where $\beta_{1}, \beta_{2}, \ldots, \beta_{5}$ are unknown regression coefficients, $\mathbf{x}$ is a vector containing the values of dummy variables ordered according to the model, and $h_{0}(t)$ is the hazard function for the reference set. That means that for $h_{0}(t)$ all dummy variables in the model are zeros, that is, $x=(0,0,0,0,0)$. When times in the continuous time model are grouped, ties in failure times can be observed. If the number of observations of ties is tolerable with respect to computing time, the unknown regression coefficients can be estimated, for example, by the Breslow result $[17,18]$ or the Efron result [19]. Generally, since effects of covariates could vary by categories, the model can be considered with interactions, which 
means that covariates can interact in affecting the hazard function. It should be noted that the quantitative variables may also be included in the Cox proportional hazards model as the explanatory variables. That means that when different rotational speeds are used in the study, rpm can be included into the model as the quantitative variable. Finally, the ratio of hazard functions, known as the hazard ratio, is a useful tool for comparing the risk of fracture between different categories of one categorical variable. The hazard ratio can be directly obtained from the Cox proportional hazards model. Due to the proportionality, the hazard ratio is the same at any time $t$. Note that the proportions of the hazard functions do not depend on the choice of the reference set in the model without interactions.

\section{Materials and Methods}

Four Ni-Ti endodontic instrument systems were tested: Wizard Navigator (Medin, Nové Město na Moravě, Czech Republic); Mtwo (Sweden and Martina, Padova, Italy); Revo$S$ (Micro-Mega, Besancon, France); and ProTaper (DentsplyMaillefer, Ballaigues, Switzerland). The ProTaper group consisted of $17 \mathrm{~F} 1$ files of size 20, 0.07 taper (yellow ISO colour) and 18 F2 files of size 25, 0.08 taper (red ISO colour); Mtwo of 17 files of size 20, 0.06 taper (yellow ISO colour) and 17 files of size 25, 0.06 taper (red ISO colour); Wizard Navigator of $20 \mathrm{~W}-3$ files of size 20, 0.06 taper (yellow ISO colour) and 20 W-4 files of size 25, 0.06 taper (red ISO colour); and Revo$S$ of 15 files of size 20, 0.04 taper (yellow ISO colour) and 18 files of size 25, 0.06 taper (red ISO colour). All instruments were new and $25 \mathrm{~mm}$ in working length. Seventy-three of NiTi rotary systems were tested in a steel block with a simulated canal with a $3 \mathrm{~mm}$ radius of curvature, and sixty-nine of $\mathrm{Ni}-\mathrm{Ti}$ rotary systems were tested in a steel block with a $5 \mathrm{~mm}$ radius of curvature and a 60-degree angle of curvature. Numbers of instruments tested in simulated canals of a specified radius of curvature according to the instruments' size are listed in Table 1. All 142 instruments had been firstly checked by a microscope for visible defects and deformations.

The cyclic fatigue-testing device was composed of a mainframe with desk and a mobile iron fixing head for an electric handpiece and stainless steel blocks with the artificial canals. The artificial canals were manufactured by precision electroerosive wire cutting to reproduce the instrument's size and taper. It provided the instrument with a suitable simulated root canal with a 60-degree angle of curvature and 3 and $5 \mathrm{~mm}$ radii of curvature (Figure 1 ).

The centre of the curvature was $6 \mathrm{~mm}$ from the tip of the instrument and the curved segment of the canal was approximately $3 \mathrm{~mm}$ in length for a $3 \mathrm{~mm}$ radius and $6 \mathrm{~mm}$ in length for a $5 \mathrm{~mm}$ radius. The length of both artificial canals was $25 \mathrm{~mm}$. The instruments were rotated at a constant speed of $250 \mathrm{rpm}$ using a 16:1 reduction handpiece (W \& H Dentalwerk, Burmoos, Austria), powered by a torque-controlled electric stepper motor (Sirona X-Smart; Dentsply Maillefer, Ballaigues, Switzerland). All instruments were rotated until fracture occurred. The time to fracture for each file was visually recorded with a 1/100 chronometer and rounded to the nearest whole second. Fractographic
TABLE 1: Numbers of four Ni-Ti rotary instruments of the size specified by ISO standards (yellow for size 20, red for size 25) tested for the canal of $3 \mathrm{~mm}$ and of $5 \mathrm{~mm}$ radius of curvature.

\begin{tabular}{cccc}
\hline Radius & System & Number & ISO colour (yellow/red) \\
\hline \multirow{4}{*}{$3 \mathrm{~mm}$} & Mtwo & 18 & $9 / 9$ \\
& Wizard Navigator & 20 & $10 / 10$ \\
& Revo-S & 17 & $7 / 10$ \\
& ProTaper & 18 & $9 / 9$ \\
\hline \multirow{4}{*}{$5 \mathrm{~mm}$} & Mtwo & 16 & $8 / 8$ \\
& Wizard Navigator & 20 & $10 / 10$ \\
& Revo-S & 16 & $8 / 8$ \\
& ProTaper & 17 & $8 / 9$ \\
\hline
\end{tabular}

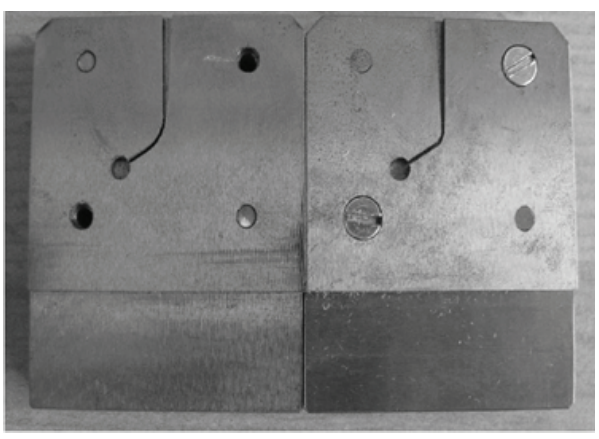

FIgURE 1: Artificial canals in stainless steel with radii of curvature of $5 \mathrm{~mm}$ (left panel) and of $3 \mathrm{~mm}$ (right panel).

analysis of the fracture surface of each file was performed by scanning electron microscope (Tescan Vega TS 5135, Czech) in order to determine mode of fracture (Figure 2). The data were analysed by the Kaplan-Meier estimator of survival function, the Cox proportional hazards model, the Wald test for significance of covariates, and the Wald test for significance of regression model.

\section{Results}

The survival functions were estimated for four studied categories of rotary systems (Figure 3), for two radii of curvature (Figure 4), and for two ISO colour-coded instrument sizes (Figure 5). The results indicate that the factors such as the variables should be considered when evaluating cyclic resistance for root canal treatment. In Figure 3, we can see that the survival function for ProTaper shows the worst survival performance, while the survival function for Mtwo demonstrates the best one. 75\% of ProTaper instruments failed within 53 seconds compared to Mtwo instruments with only $25 \%$ failing within 94 seconds. The survival functions of Wizard Navigator instruments and Revo-S instruments are close to each other. Our results show that $50 \%$ of Wizard Navigator instruments failed within 103 seconds, while $50 \%$ of Revo-S instruments failed within 75 seconds. From Figure 4 it follows that the survival function for the instruments rotated in the artificial canal with a $3 \mathrm{~mm}$ radius of curvature decreases faster than the survival function for the instruments rotated in the artificial canal with a $5 \mathrm{~mm}$ radius 


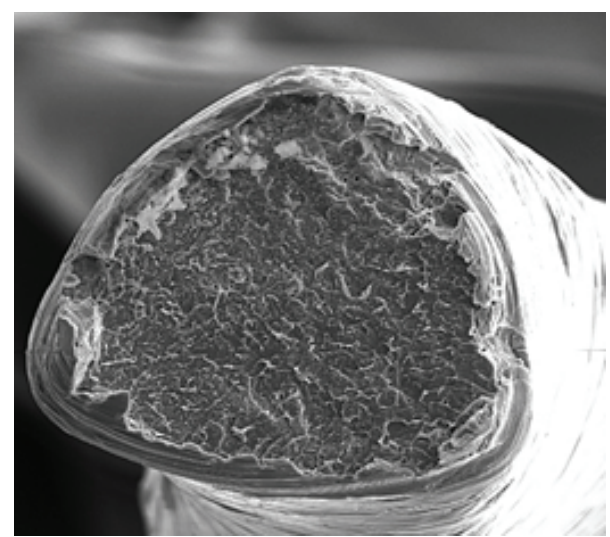

FIGURE 2: Fractographic analysis of the fracture surface of a Wizard Navigator instrument after cyclic fatigue testing (magnification 400x).

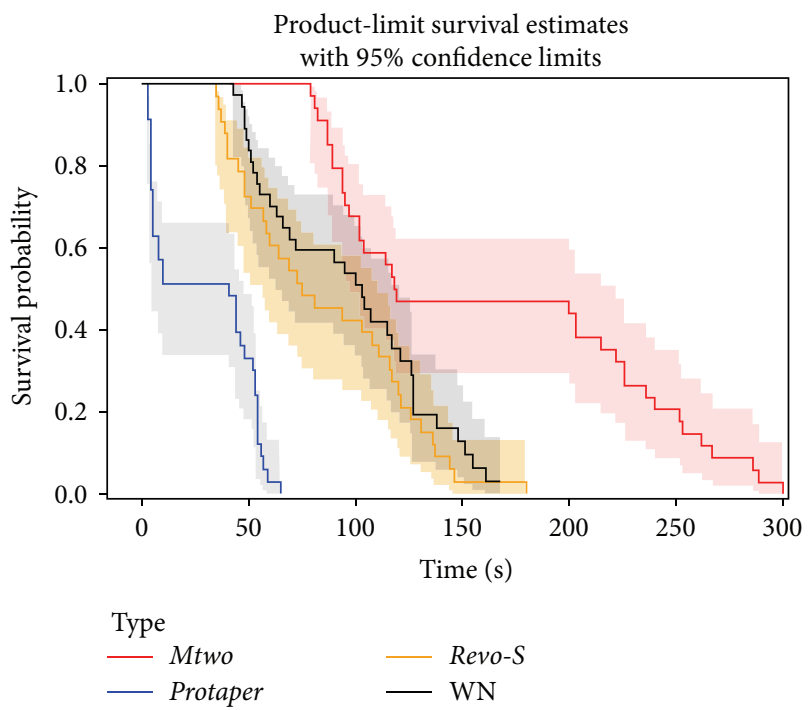

FIgUre 3: The Kaplan-Meier estimate of the survival function (probability of cyclic resistance) of rotary instruments categorized by the type of rotary instrument.

of curvature. $50 \%$ of instruments failed within 54 seconds in the canals with a $3 \mathrm{~mm}$ radius, whereas only $25 \%$ failed within 73 seconds in the canals with a $5 \mathrm{~mm}$ radius. The results demonstrated in Figure 5 indicate that the instruments marked by red colour in ISO colour system show a worse survival performance, as the time to the cyclic fatigue fracture is significantly shorter than for the instruments marked by yellow colour. $50 \%$ of red ISO coded rotary instruments failed within 73 seconds, while $50 \%$ of yellow ISO coded instruments failed within 90 seconds. Since the estimated survival functions do not cross, the statistical significance of the factor is determined by the Cox proportional hazards model (Table 2).

The exponentiated estimated coefficients from Table 2 are interpretable as multiplicative effects on the hazard. Thus, the proportion of the hazard function for a $5 \mathrm{~mm}$ radius and the hazard function for a $3 \mathrm{~mm}$ radius of the cyclic

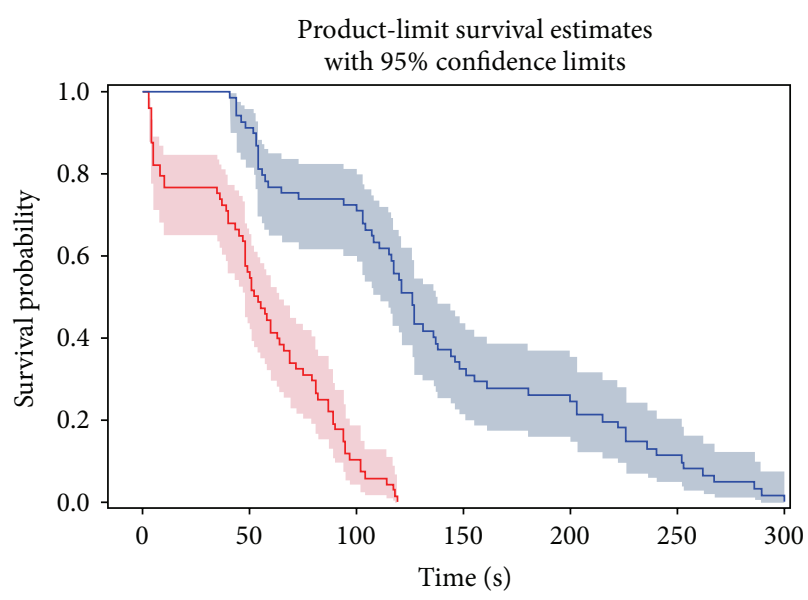

Radius

$$
\begin{array}{r}
3 \mathrm{~mm} \\
-5 \mathrm{~mm}
\end{array}
$$

FIgURE 4: The Kaplan-Meier estimate of the survival function (probability of cyclic resistance) of rotary instruments categorized by the radius of canal curvature.

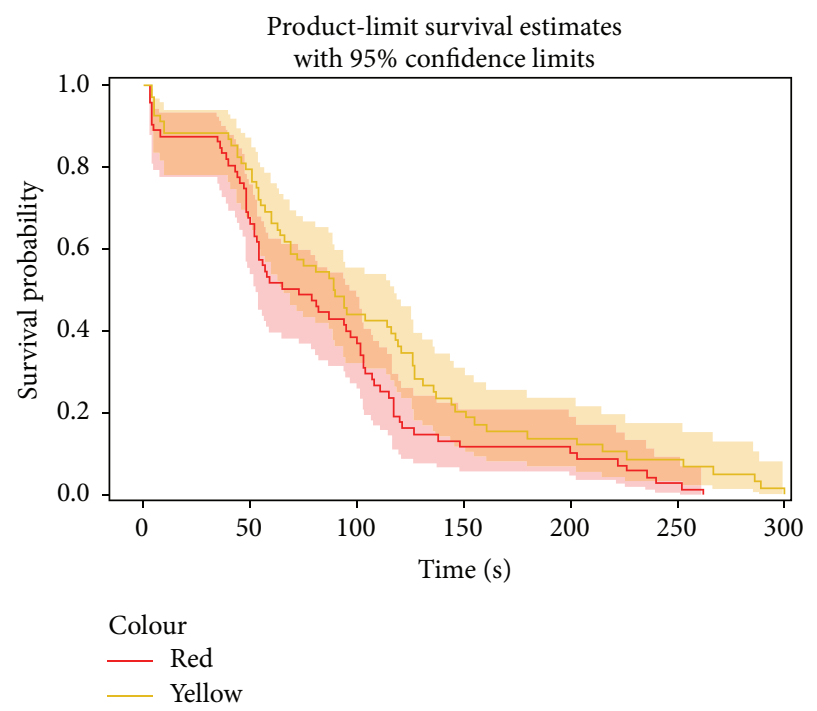

FIGURE 5: The Kaplan-Meier estimate of the survival function (probability of cyclic resistance) of rotary instruments categorized by the size coding by ISO standards (yellow for size 20, red for size 25).

fatigue fracture is $\exp \left\{\widehat{\beta}_{1}\right\}=\exp \{-5.461\}=0.004$. It means that the hazard of the cyclic fatigue fracture of $\mathrm{Ni}$ $\mathrm{Ti}$ instruments during the preparation of the canal with a $5 \mathrm{~mm}$ radius is 0.004 times less than for a $3 \mathrm{~mm}$ radius, or equivalently, the hazard of failure for a $3 \mathrm{~mm}$ radius is $1 / 0.004$ $=250$ times higher than for the simulated canal with a $5 \mathrm{~mm}$ radius of curvature. The proportion of the hazard function for the yellow ISO colour coded instruments and the red ISO colour instruments is $\exp \left\{\widehat{\beta}_{5}\right\}=\exp \{-1.7\}=0.31$. It means that, for the red ISO coloured instruments, the hazard of the cyclic fatigue is $1 / 0.31=3.226$ times higher than for the yellow ISO coloured ones. The risk of cyclic 
TABLE 2: Results of the Cox proportional hazard model of the cyclic fatigue lifetime of the instruments. The set of reference categories is $3 \mathrm{~mm}$ radius, Wizard Navigator, and red ISO colour.

\begin{tabular}{lcccc}
\hline & Estimat. $\beta$ & Stand. error & $p$ value & Hazard ratio \\
\hline $5 \mathrm{~mm}$ & -5.461 & 0.476 & 0.0001 & 0.004 \\
Mtwo & -3.212 & 0.394 & 0.0001 & 0.040 \\
ProTaper & 5.901 & 0.566 & 0.0001 & 365.291 \\
Revo-S & 0.583 & 0.259 & 0.0250 & 1.791 \\
yellow & -1.700 & 0.207 & 0.0001 & 0.310 \\
\hline
\end{tabular}

fatigue for ProTaper files is $\exp \left\{\widehat{\beta}_{2}\right\}=\exp \{5.901\}=365.291$ higher than for Wizard Navigator files, and Wizard Navigator instruments are $1 / 1.791=0.558$ times more resistant than Revo-S files. Mtwo instruments are most resistant to cyclic fatigue, with the hazard of failure 0.040 timesless than for Wizard Navigator instruments. While Mtwo files have been found significantly most resistant ( $p$ value $<0.001$ ), ProTaper files have been found significantly least resistant to cyclic fatigue of all four tested rotary systems ( $p$ value $<0.001$ ). A statistically significant difference has been found also between Wizard Navigator and Revo-S instruments ( $p$ value $=0.025)$. The significance of covariates was verified by the Wald test, wherein 0.001 level for all 5 covariates was reached (Table 2, column 4). The omnibus test of the model was tested by the Wald test, which showed that the $p$ values were less than 0.001. All computations were done by the statistical software SAS Version 9.3 (SAS Institute, NC, USA).

\section{Discussion}

The aim of this study was to validate the survival analysis assessing the cyclic fatigue resistance of different Ni-Ti rotary system. The effect of the type of rotary system, radius of curvature, and instrument size on the cyclic fatigue resistance of $\mathrm{Ni}$ - $\mathrm{Ti}$ instruments was analysed by the Cox proportional hazards model. The results of the present study revealed that Mtwo instruments resisted fatigue fracture for the longest time. On the other hand, ProTaper showed significantly lesser resistance times than the other instruments. Wizard Navigator files were found to be significantly more resistant than Revo-S files. Both the radius of curvature and the instrument size were found to be significant factors in determining fatigue resistance of the files. Previous studies showed that the time to failure decreases with the decreasing radius of curvature and the angle of the curvature greater than 30 degrees $[1,2,7]$. The results of our investigation confirmed that instrument life span is inversely proportional to the instrument size $[2,7,20]$. The influence of the curved segment length of an artificial canal on fatigue resistance was detected in [5].

The continuous cycle of tensile and compressive forces in the area of curvature of the canal to which Ni-Ti rotary instruments are subject produces a destructive form of loading [2]. Prolonged use of engine-driven instruments would consequently increase the risk of instrument distortion and/or separation. Therefore, manufacturers state that the only predictable way to prevent failure is to discard rotary files regularly after a certain number of uses. Still, there is no consensus or scientific evidence as to the exact number of uses before the files should be discarded. Moreover, NiTi instruments can undergo fracture within their elastic limit without any visible sign of previous permanent deformation $[2,3]$. According to many practitioners, instruments should be disposed of after a single use in very complex, calcified, and curved canals. The survival function is fundamental to a survival analysis. Using the Kaplan-Meier estimate of survival function, we estimated the probability that an instrument resists over a specified time $t$. Note that the times recorded for the instrument with torsional fracture can also be included in the analysis. Such observations would be considered as rightcensored and all used procedures are suitable for the rightcensored [21] data as well.

A survival analysis is a very useful tool in clinical research, providing invaluable information about an intervention. There are books available which provide basic knowledge about the theory, as well as its application and its interpretation [22]. In addition, both the Kaplan-Meier estimator of the survival function and the Cox proportional hazards model, as well as the relevant tests, are included in most analytical software.

\section{Conclusion}

We have evaluated the effect of the type of rotary system (Wizard Navigator, Mtwo, Revo-S, and ProTaper), the radius of curvature of an artificial canal ( 3 and $5 \mathrm{~mm}$ ), and the instrument size (ISO colour coded: yellow for size 20 and red for size 25) on the time to the cyclic fatigue fracture. The resistance to failure is strongly affected by the type of rotary system. Mtwo files performed significantly better than all the other files. The highest hazard of the cyclic fatigue fracture was registered for ProTaper instruments. Wizard Navigator files were found to be significantly more resistant than Revo-S files. The failure time is also strongly affected by the radius of curvature. In particular, an instrument's life span is directly proportional to the radius of curvature. The size of an instrument was also found to be a significant factor in determining fatigue resistance of the files, with resistance to failure being inversely proportional to the instrument size.

\section{Conflict of Interests}

The authors declare that there is no conflict of interests regarding the publication of this paper.

\section{Acknowledgment}

The authors thankfully acknowledge the support given by the Operational Program Education for Competitiveness, European Social Fund (Projects CZ.1.07/2.3.00/20.0170 and CZ.1.07/2.3.00/30.0041 of the Ministry of Education, Youth and Sports of the Czech Republic). 


\section{References}

[1] N. M. Grande, G. Plotino, R. Pecci, R. Bedini, V. A. Malagnino, and F. Somma, "Cyclic fatigue resistance and three-dimensional analysis of instruments from two nickel-titanium rotary systems," International Endodontic Journal, vol. 39, no. 10, pp. 755763, 2006.

[2] J. P. Pruett, D. J. Clement, and D. L. Carnes Jr., "Cyclic fatigue testing of nickel-titanium endodontic instruments," Journal of Endodontics, vol. 23, no. 2, pp. 77-85, 1997.

[3] B. Sattapan, G. Nervo, J. Palamara, and H. Messer, "Defects in nickel titanium endodontic rotary files after clinical usage," Journal of Endodontics, vol. 26, no. 3, pp. 161-165, 2000.

[4] T. P. Serene, J. D. Adams, and A. Saxena, Nickel-Titanium Instruments: Applications in Endodontics, Ishiyaku EuroAmerica, St. Louis, Mo, USA, 1995.

[5] H. P. Lopes, E. J. L. Moreira, C. N. Elias, R. A. de Almeida, and M. S. Neves, "Cyclic fatigue of Protaper instruments," Journal of Endodontics, vol. 33, no. 1, pp. 55-57, 2007.

[6] B. Martín, G. Zelada, P. Varela et al., "Factors influencing the fracture of nickel-titanium rotary instruments," International Endodontic Journal, vol. 36, no. 4, pp. 262-266, 2003.

[7] Y. Haïkel, R. Serfaty, G. Bateman, B. Senger, and C. Allemann, "Dynamic and cyclic fatigue of engine-driven rotary nickeltitanium endodontic instruments," Journal of Endodontics, vol. 25, no. 6, pp. 434-440, 1999.

[8] P. Parashos, I. Gordon, and H. H. Messer, "Factors influencing defects of rotary nickel-titanium endodontic instruments after clinical use," Journal of Endodontics, vol. 30, no. 10, pp. 722-725, 2004.

[9] J. T. McSpadden, "Mastering the technique," in Mastering Endodontic Instrumentation, J. T. McSpadden, Ed., pp. 105-124, Cloudland Institute, Chattanooga, Tenn, USA, 2007.

[10] G. S. Cheung, "Instrument fracture: mechanisms, removal of fragments, and clinical outcomes," Endodontic Topics, vol. 16, pp. 1-26, 2009.

[11] E. Berutti, G. Chiandussi, I. Gaviglio, and A. Ibba, "Comparative analysis of torsional and bending stresses in two mathematical models of nickel-titanium rotary instruments: ProTaper versus ProFile," Journal of Endodontics, vol. 29, no. 1, pp. 15-19, 2003.

[12] M. T. Biz and J. A. P. Figueiredo, "Morphometric analysis of shank-to-flute ratio in rotary nickel-titanium files," International Endodontic Journal, vol. 37, no. 6, pp. 353-358, 2004.

[13] Y. L. Turpin, F. Chagneau, and J. M. Vulcain, "Impact of two theoretical cross-sections on torsional and bending stresses of nickel-titanium root canal instrument models," Journal of Endodontics, vol. 26, no. 7, pp. 414-417, 2000.

[14] E.-W. Zhang, G. S. P. Cheung, and Y.-F. Zheng, "Influence of cross-sectional design and dimension on mechanical behavior of nickel-titanium instruments under torsion and bending: a numerical analysis," Journal of Endodontics, vol. 36, no. 8, pp. 1394-1398, 2010.

[15] E. L. Kaplan and P. Meier, "Nonparametric estimation from incomplete observations," Journal of the American Statistical Association, vol. 53, pp. 457-481, 1958.

[16] D. R. Cox, "Regression models and life-tables," Journal of the Royal Statistical Society Series B: Methodological, vol. 34, no. 2, pp. 187-220, 1972.

[17] N. E. Breslow, “Discussion of professor Cox’s paper," Journal of the Royal Statistical Society, vol. 34, pp. 216-217, 1972.

[18] N. E. Breslow, "Covariance analysis of censored survival data," Biometrics, vol. 30, no. 1, pp. 89-99, 1974.
[19] B. Efron, “The efficiency of Cox's likelihood function for censored data," Journal of the American Statistical Association, vol. 72, no. 359, pp. 557-565, 1977.

[20] D. N. Dederich and K. L. Zakariasen, "The effects of cyclical axial motion on rotary endodontic instrument fatigue," Oral Surgery, Oral Medicine, Oral Pathology, vol. 61, no. 2, pp. 192196, 1986.

[21] J. P. Klein and M. L. Moeschberger, Survival Analysis: Techniques for Censored and Truncated Data, Springer, New York, NY, USA, 1997.

[22] D. G. Kleinbaum and M. Klein, Survival Analysis: A SelfLearning Text, Springer, New York, NY, USA, 2nd edition, 2005. 

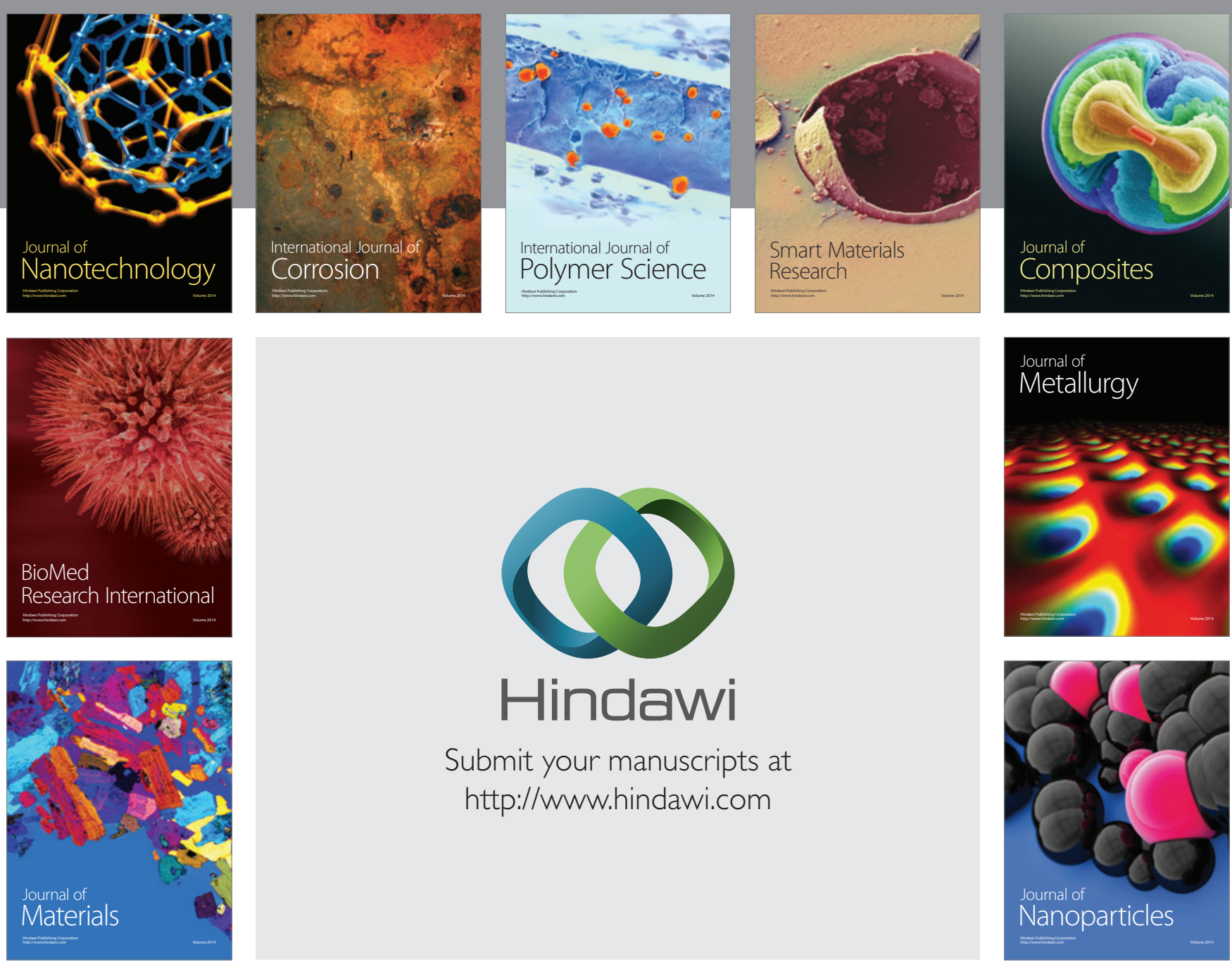

Submit your manuscripts at http://www.hindawi.com
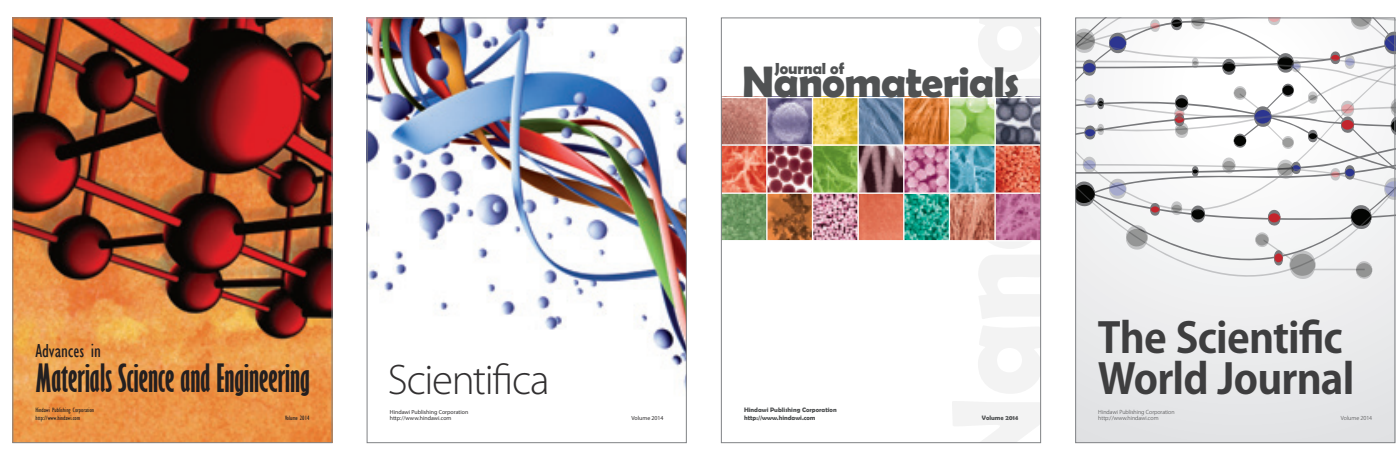

\section{The Scientific World Journal}
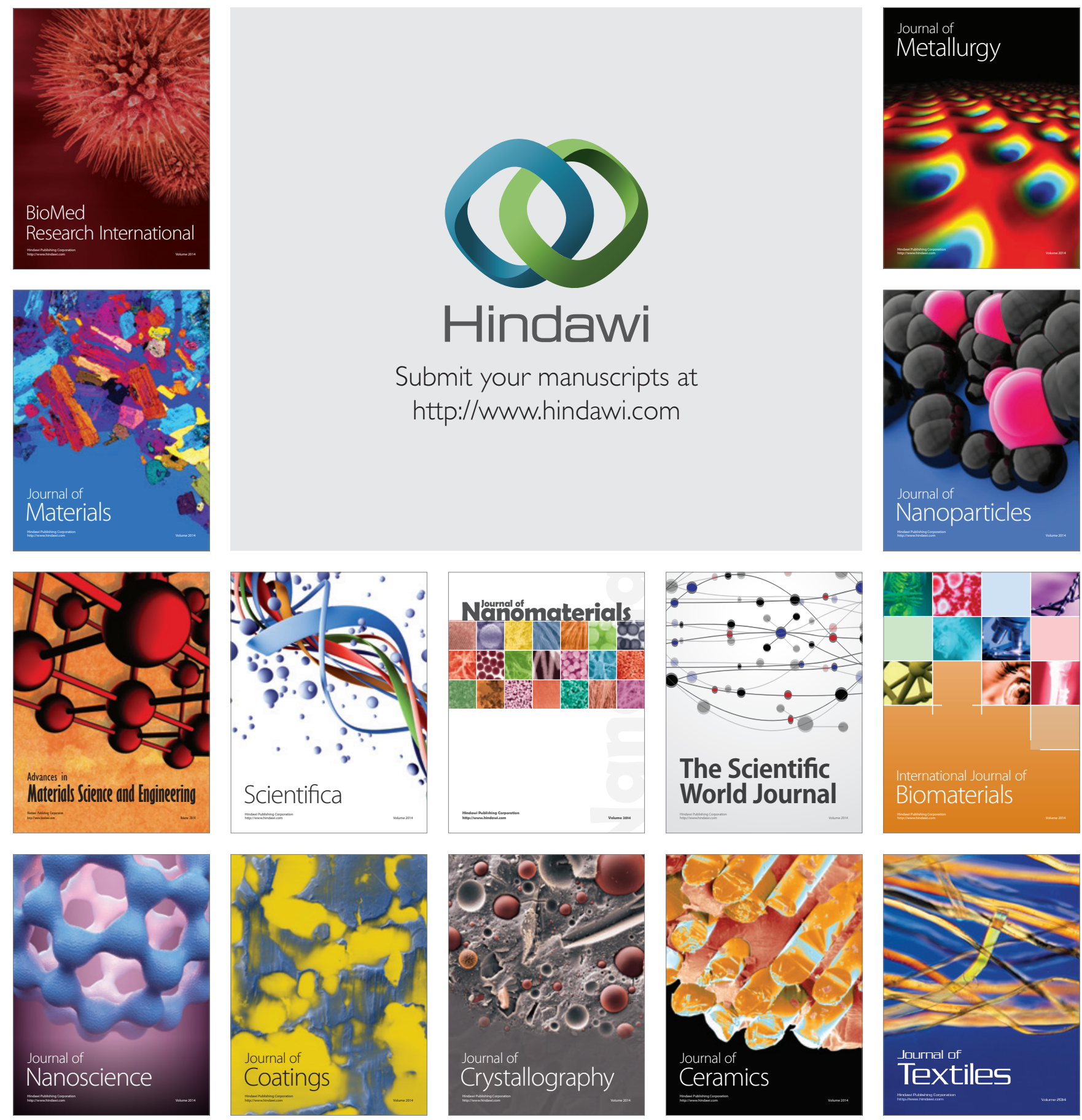